\title{
Patchy Core/Shell, Magnetite/Silver Nanoparticles via Green and Facile Synthesis: Routes to Assure Biocompatibility
}

\author{
Carlos M. Ramírez-Acosta ${ }^{1}{ }^{1}$, Javier Cifuentes ${ }^{2}{ }^{(\mathbb{D}}$, Juan C. Cruz ${ }^{2,3, *(\mathbb{D})}$ and Luis H. Reyes ${ }^{1, *(1)}$ \\ 1 Grupo de Diseño de Productos y Procesos (GDPP), Department of Chemical and Food Engineering, \\ Universidad de los Andes, Bogotá 111711, Colombia; cm.ramirez10@uniandes.edu.co \\ 2 Department of Biomedical Engineering, Universidad de los Andes, Bogotá 111711, Colombia; \\ jf.cifuentes10@uniandes.edu.co \\ 3 School of Chemical Engineering and Advanced Materials, The University of Adelaide, \\ South Australia 5005, Australia \\ * Correspondence: juan.cruz@adelaide.edu.au (J.C.C.); lh.reyes@uniandes.edu.co (L.H.R.); \\ Tel.: +57-1-3394949 (ext. 1789) (J.C.C.); +57-1-3394949 (ext. 1702) (L.H.R.)
}

Received: 1 September 2020; Accepted: 14 September 2020; Published: 17 September 2020

\begin{abstract}
Nanomedicine is entering a high maturity stage and is ready to reach full translation into the clinical practice. This is because of the ample spectrum of applications enabled by a large arsenal of nanostructured materials. In particular, bimetallic patchy core/shell nanoparticles offer tunable surfaces that allow multifunctional responses. Despite their attractiveness, major challenges regarding the environmental impact and biocompatibility of the obtained materials are yet to be solved. Here, we developed a green synthesis scheme to prepare highly biocompatible patchy core/shell magnetite/silver nanoparticles for biological and biomedical applications. The magnetite core was synthesized by the co-precipitation of ferric chloride and ferrous chloride in the presence of $\mathrm{NaOH}$. This was followed by the patchy silver shell's growth by a green synthesis approach based on natural honey as a reducing agent. A purification process allowed selecting the target patchy nanoparticles and removing excess toxic reagents from the synthesis very efficiently. The obtained patchy magnetite/silver nanoparticles were characterized by UV-Vis spectrophotometry, dynamic light scattering (DLS), thermogravimetric analysis (TGA), Fourier transform infrared spectroscopy (FTIR), scanning electron microscope equipped with energy-dispersive spectroscopy (SEM + EDS), and transmission electron microscopy (TEM). The morphology, patchiness level, and size of the nanoparticles were determined via SEM and TEM. In addition, the spectrophotometric characterization confirmed the presence of the patchy silver coating on the surface of the magnetite core. The nanoparticles show high biocompatibility, as evidenced by low cytotoxicity, hemolytic effect, and platelet aggregation tendency. Our study also provides details for the conjugation of multiples chemistries on the surface of the patchy bimetallic nanoparticles, which might be useful for emerging applications in nanomedicine, where high biocompatibility is of the utmost importance.
\end{abstract}

Keywords: patchy nanoparticles; core/shell; green synthesis; biocompatibility

\section{Introduction}

Nanomedicine is currently approaching the maturity level required to translate into the clinical practice fully. This has been attributed to our increasing ability to synthesize and modify objects at the nanoscale with relatively high precision [1-3]. As a result, numerous applications ranging from biosensors to drug and gene delivery now hold much promise to impact patients' health positively $[1,2]$. One example of complex nanostructured objects with potential nanomedicine 
applications is bimetallic nanoparticles, combining attractive features of two different metallic elements arranged in unique supramolecular structures [4-6]. Examples include bimetallic nanoparticles combining nickel and cobalt, and silver and platinum $[7,8]$. In the first case, the nanostructure was formed by the microwave irradiation synthesis method and exploited the magnetism of nickel and the solid and liquid solubility of cobalt in a core/shell arrangement [7]. In contrast, in the second case, the main properties were antioxidant and reactive oxygen species, and the formed silver-platinum structure was obtained by a green synthesis [8]. Finally, another patchy core/shell system for nanomedicine applications is magnetite/titanium oxide, which has proven useful in the highly selective enrichment of phosphopeptides [9].

Patchy core/shell nanoparticles have attracted significant attention, mainly due to their tunable surface and the properties of both the core and metallic shells [4,10]. Features such as magnetism and chemical compatibility can be combined relatively straightforwardly, thereby making them of great interest in nanomedicine applications such as antibacterial systems, imaging agents, and drug delivery carriers $[4,10,11]$. Moreover, they can be functionalized with different macromolecules, including polymers, peptides, antibodies, and even nucleic acids [12,13]. For instance, the obtained nanoconjugates have found applications in the ultrasensitive detection of biological species or the targeted delivery of nucleic acids at the subcellular level [14,15]. Most patchy core/shell nanoparticles' synthesis methods mainly rely on bottom-up approaches where chemical precursors can self-assemble into the desired arrangement in multi-stage processes with controlled conditions [16-19]. Materials obtained by this method generally exhibit soft units with flexible bonding patterns and sizes in the range of 1 to $100 \mathrm{~nm}$. Top-down routes are not as popular mainly because the resulting materials exhibit pre-defined and fixed patchiness, and they rely on sophisticated equipment for manufacturing and processing $[17,19,20]$. Some of the bottom-up synthesis schemes include electrochemical, sonochemical, and thermal decomposition, which require high energy levels and controlled conditions such as inert atmospheres [21-23]. For example, the electrochemical synthesis to manufacture silver shells on gold nanoparticles and the sonochemical route to synthesize magnetite core with a deposition of superficial silica [21-23].

Compared to conventional synthesis schemes, the greener ones mainly encompass a growth media or a natural organic compound [24,25]. Consequently, during the shell or coating formation, the crude extract's quality assurance plays a major role, as it defines the functional groups interacting, which ultimately controls the level of patchiness and the homogeneity of the final nanomaterial. Several synthesis methods produce the patchy core/shell made of metal/metal systems, including ligand selection, direct and self-assembly, physical or chemical absorption, and seeded growth $[11,25]$. Due to its flexibility, versatility, and relatively low costs, seeded-growth schemes have been extensively implemented $[19,26]$. However, the need for environmentally friendly and readily accessible synthesis methods led to greener versions of previously mentioned synthesis schemes [11,24,25]. As a result, novel green synthesis routes have been successfully developed to manufacture core/shell bimetallic nanoparticles, such as gold/silver and magnetite/silver [24,27,28]. Moreover, most studies report methodologies involving anaerobic conditions or drying out the final nanomaterial to remove residues of the natural extract $[23,25]$. Thus, the purification process represents one of the main challenges regarding the full potential of extract-based green syntheses.

Therefore, this study is dedicated to developing a facile method to synthesize patchy core/shell magnetite/silver nanoparticles, starting for magnetite cores as seeds and followed by a green synthesis of the patchy silver shell $[29,30]$. In addition, the patchy core/shell system proposed here focuses on a tunable surface as an advantage for biomedical technologies where multiple chemistries and functional groups might be required. The obtained materials exhibit properties similar to those described by Haiyang et al. in their review about core/shell nanoparticles [31]. We also described a purification process to assure that potentially cytotoxic chemical species are entirely removed from the nanoparticle suspensions. Moreover, the nanoparticles were characterized via electron microscopy and thermal and spectroscopic techniques. Our study also provides details for the conjugation of multiples chemistries 
on the surface of the patchy bimetallic nanoparticles, which might be useful for emerging applications in nanomedicine, where high biocompatibility is of the utmost importance.

\section{Materials and Methods}

\subsection{Synthesis and Characterization of the Magnetite Core}

The synthesis was conducted via the co-precipitation method using iron salts as a precursor. Iron (II) chloride and iron (III) chloride were purchased from J. T. Baker (USA) and Merck (Kenilworth, NJ, USA), respectively. Briefly, a 2 Fe(II):1 Fe(III) molar ratio solution was added dropwise to a sodium hydroxide solution $5 \mathrm{M}$ at $90{ }^{\circ} \mathrm{C}$ (PanReac AppliChem, Darmstadt, Germany) at a rate of $5 \mathrm{~mL} / \mathrm{min}$, as shown in Figure 1: Magnetite synthesis. Mechanical agitation was kept at $400 \mathrm{RPM}$ with a Rushton impeller in a $600 \mathrm{~mL}$ beaker, while the iron solutions were added. An ultrasonic bath was used to resuspend the synthesized nanoparticles. The excess reagents left from an incomplete reaction were removed, in the first place, by placing a neodymium magnet below a precipitation beaker, thereby accelerating the magnetic nanoparticles precipitation. Secondly, the supernatant was removed and discarded, and finally, water at approximately $75^{\circ} \mathrm{C}$ was added, followed by resuspension in an ultrasonic bath. The procedure was repeated several times until the supernatant was colorless (Figure 1: Purification). Finally, the washed nanoparticles were resuspended in Milli-Q water. Bare magnetite nanoparticles were imaged via scanning electron microscope (SEM, TESCAN LYRA3 FIB-SEM, Czech Republic) and subsequently characterized by UV-Vis spectrophotometry (Thermo Scientific GENESYS, Waltham, MA, USA). Dynamic light scattering (DLS) was used to determine the hydrodynamic diameter (Zeta-Sizer Nano-ZS, Malvern Panalytical, Malvern, UK). The UV-Vis absorbance spectra were collected for a suspension of the nanoparticles in Type-I water $(0.1 \mathrm{mg} / \mathrm{mL})$ from 200 to $1000 \mathrm{~cm}^{-1}$.

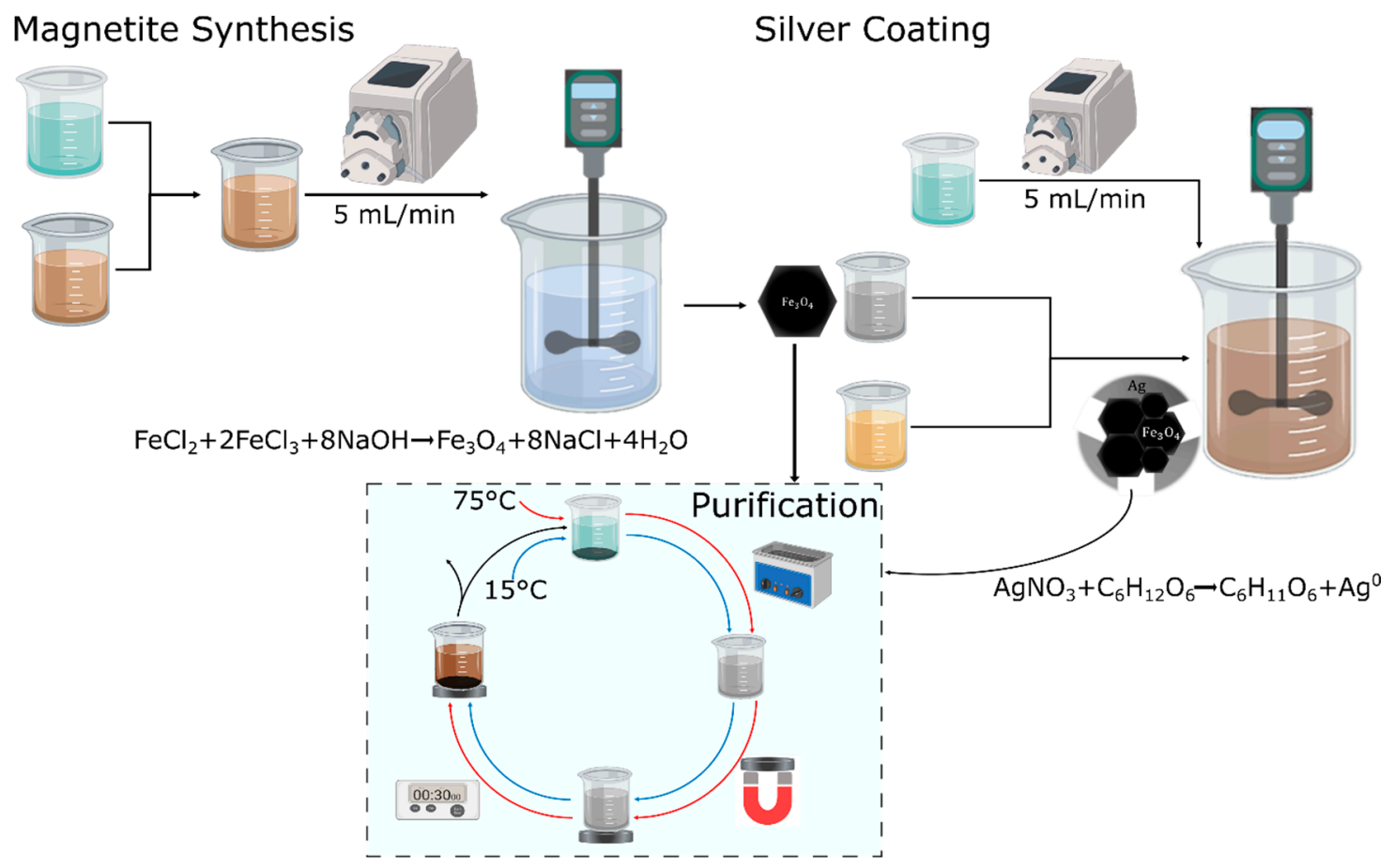

Figure 1. Schematic for the green synthesis of patchy core/shell, magnetite/silver nanoparticles. First, magnetite was synthesized using the co-precipitation method (the reaction scheme taking place is also shown). Second, the patchy silver coating was deposited by reducing silver nitrate in a honey solution (reducing agent) with the glucose present (reaction shown below). Finally, the dotted frame process represents the cleaning or purification steps with either hot $\left(75^{\circ} \mathrm{C}\right)$ or cold water $\left(15^{\circ} \mathrm{C}\right)$. 


\subsection{Silver Shell Formation}

A redox reaction, starting with $1 \mathrm{mM}$ silver nitrate (Merck, Kenilworth, NJ, USA) and 20\% (w/v) aqueous honey solution from a local farmer's market (as a reducing agent), was used to create the silver shell via the developed green deposition scheme. The procedure consists in dissolving $5 \mathrm{~mL}$ of magnetite $(1 \mathrm{mg} / \mathrm{mL})$ in the honey solution followed by pumping the silver nitrate into the suspension at $5 \mathrm{~mL} / \mathrm{min}$. Magnetite structural integrity can be compromised when $\mathrm{pH}$ descends below 4 , and for that reason, it was maintained above 4 with the aid of $\mathrm{NaOH}$. Once the silver nitrate solution was added, the $\mathrm{pH}$ was adjusted to 8 using a $\mathrm{NaOH}$ solution (1M). The experimental setup for the synthesis is shown in Figure 1: Silver Coating. Once the silver coating was completed, the obtained nanoparticles were suspended in an organic compounds' solution remaining from the synthesis. Commercially available (from a local producer) honey's organic compounds include acetic acid, citric acid, vitamin B, and glucose. The obtained nanomaterials were most likely a mixture of uncoated magnetite, free silver nanoparticles, and the patchy magnetite/silver nanoparticles.

\subsection{Magnetite/Silver Purification and Physical Characterization}

In order to purify the patchy magnetite/silver nanoparticles, free silver nanoparticles were separated from magnetite and magnetite/silver via magnetic precipitation with the aid of a neodymium magnet by following the cleaning steps described above (Figure 1: Purification). Then, the remaining bare magnetite was precipitated using hydrochloric acid $(1 \mathrm{mM})$ until the $\mathrm{pH}$ reached 3 . Under such conditions, magnetite nanoparticles change their crystalline structure and tend to form an iron salt, which lacks magnetic properties. In contrast, under the same conditions, the patchy magnetite/silver nanoparticles preserve their magnetic responsiveness and can be separated magnetically, as discussed above in the magnetite synthesis section (Figure 1: Purification). The obtained magnetite/silver nanoparticles were first characterized by determining their hydrodynamic radii via DLS. In addition, the presence of the silver shell was verified by collecting the absorbance spectrum from 200 to $1000 \mathrm{~cm}^{-1}$ and comparing it with that of magnetite, which is aided by a UV-Vis spectrophotometer. The nanoparticles of magnetite and magnetite/silver were imaged, and their atomic composition was determined using a scanning electron microscope (SEM + EDS, TESCAN LYRA3 FIB-SEM, Czech Republic), which was equipped with energy-dispersive spectroscopy. A transmission electron microscope (TEM) Tecnai F30 (FEI Company, Fremont, CA, USA), at a resolution of $134 \mathrm{eV}$ and reference energy of $5.9 \mathrm{keV}$, was used to analyze the magnetite/silver coverage.

\subsection{Magnetite/Silver Surface Chemistry}

In order to evaluate the chemical reactivity of both surfaces, magnetite and silver, two different molecules were conjugated: first, an organosilane on the uncovered magnetite, and second, a cationic polymer on the silver shell. The organosilane (3-aminopropyl) triethoxysilane (APTES, 98\%, Sigma-Aldrich, St. Louis, MO, USA), a molecule commonly used to anchor on the surface of the magnetite, was conjugated by the addition of $200 \mu \mathrm{L}$ of APTES to suspensions of magnetite and magnetite/silver nanoparticles. The conjugation proceeded with sonication at $20 \mathrm{kHz}$ for $30 \mathrm{~min}$, using a sonic tip. To finalize the conjugation, the APTES that was not covalently attached to the nanomaterial surface was removed by following the purification process mentioned earlier, where water was added at approximately $75^{\circ} \mathrm{C}$, followed by magnetic precipitation (Figure 1: Purification). On the silver shell surface, the cationic polymer poly(2-dimethylamino)ethyl methacrylate) methyl chloride quaternary salt (pDMAEMA) purchased from Sigma-Aldrich (USA) was conjugated. First, the polymer was dissolved in an aqueous solution $(10 \mathrm{mg} / \mathrm{mL})$. The purified magnetite/silver nanoparticles were stirred at $500 \mathrm{RPM}$ and $50^{\circ} \mathrm{C}$ for $1 \mathrm{~h}$. With the chlorine groups left from the purification process, a Hofmann elimination reaction was carried out to conjugate the pDMAEMA. The conjugation of APTES and pDMAEMA was verified using Fourier transform infrared spectroscopy (FTIR) in a Bruker Alpha II FTIR Eco-ATR instrument (Bruker, Billerica, MA, USA) by collecting the 
spectra from 900 to $4000 \mathrm{~cm}^{-1}$ and comparing them directly to the patchy bimetallic nanoparticles. Before collecting the data, once again, the nanoparticles were purified using magnetic precipitation (Figure 1: Purification). Thermogravimetric analyses (TGA), in a simultaneous TGA/DSC instrument (TA Instruments, New Castle, DE, USA), were conducted to estimate the pDMAEMA and APTES conjugation efficiencies, implementing a linear temperature ramp at a rate of $10^{\circ} \mathrm{C} / \mathrm{min}$ from 25 to $600{ }^{\circ} \mathrm{C}$ under an inert atmosphere.

\subsection{Biocompatibility}

The hemolytic activity of magnetite and magnetite/silver bimetallic nanoparticles was tested on erythrocytes isolated from a healthy human donor's freshly drawn blood. The erythrocytes were collected by centrifuging the blood at 1800 RPM for $5 \mathrm{~min}$, discarding the plasma, and finally washing them five times with $\mathrm{NaCl}$ solution $(0.9 \%(w / v))$ and once with PBS (Phosphate Buffered Saline) (1X). An erythrocytes stock was prepared by adding $1 \mathrm{~mL}$ of isolated erythrocytes $\left(4.3 \times 10^{6}\right.$ erythrocytes $\left./ \mu \mathrm{L}\right)$ in $9 \mathrm{~mL}$ of PBS (1X). Serial dilutions of the magnetite and the patchy magnetite/silver nanoparticles (200 to $12.5 \mu \mathrm{g} / \mathrm{mL}$ ) were prepared by mixing initial stocks with PBS (1X). Triton X-100 (1\% $(v / v)$ ) and PBS (1X) were used as positive and negative controls, respectively. Erythrocytes $(100 \mu \mathrm{L})$ were seeded in a 96-well microplate, exposed to each treatment $(100 \mu \mathrm{L})$, incubated at $37^{\circ} \mathrm{C}, 5 \% \mathrm{CO}_{2}$ for one hour, and centrifuged at $1800 \mathrm{RPM}$ for $5 \mathrm{~min}$. Then, $100 \mu \mathrm{L}$ of each supernatant was read at $450 \mathrm{~nm}$ in a microplate reader. The hemolysis percentage was calculated using Equation (1).

$$
\text { Hemolysis percentage }=\frac{A b s_{450}(\text { sample })-A b s_{450}(\text { negative control })}{A b s_{450}(\text { positive control })-A b s_{450}(\text { negative control })} \times 100
$$

The platelet aggregation tendency of magnetite and patchy magnetite/silver nanoparticles was tested on platelets isolated from a healthy human donor's freshly drawn blood. A vacutainer tube was used to collect the blood sample containing sodium citrate as an anticoagulant. A platelet-rich plasma (PRP) was obtained by centrifuging the sample at $1000 \mathrm{RPM}$ for $15 \mathrm{~min}$ at room temperature $\left(25^{\circ} \mathrm{C}\right)$. Serial dilutions of the magnetite and magnetite/silver nanoparticles (200 to $12.5 \mu \mathrm{g} / \mathrm{mL}$ ) were prepared by mixing concentrated stocks with PBS (1X). As a positive control, thrombin (6 U) was used, and PBS (1X) served as a negative reference. Then, $50 \mu \mathrm{L}$ of PRP was mixed with $50 \mu \mathrm{L}$ of the different samples in a 96-well microplate and incubated at $37^{\circ} \mathrm{C}, 5 \% \mathrm{CO}_{2}$ for $5 \mathrm{~min}$. Finally, a sample of $50 \mu \mathrm{L}$ of each supernatant was extracted, and absorbance was read at $620 \mathrm{~nm}$ in a microplate reader.

The quantification of the lactate dehydrogenase enzyme (LDH), using a commercially available Cytotoxicity Detection Kit (LDH) (Roche, Basel, Switzerland), was used to determine the cytotoxicity of purified patchy magnetite/silver nanoparticles. The cytotoxicity was tested by exposing the samples to Vero cells (ATCC ${ }^{\circledR}$ CCL-81) at a cell density of 100,000 cells/mL. Serial dilutions (i.e., 200-12.5 $\mu \mathrm{g} / \mathrm{mL}$ ) were prepared by mixing concentrated stocks with DMEM (Dulbecco's Modified Eagle's medium) media. Triton X-100 (1\% (v/v)) was used as positive control, and DMEM media was used as the negative one. Then, $100 \mu \mathrm{L}$ of the cell stock (DMEM, supplemented with 10\% (v/v) Fetal Bovine Serum-FBS) were deposited in 96-well microplates (10,000 cells/well) and incubated at $37^{\circ} \mathrm{C}, 5 \% \mathrm{CO}_{2}$ for $24 \mathrm{~h}$. After incubation, culture media was removed, and cells were washed with PBS (1X). Later, PBS was removed, and $100 \mu \mathrm{L}$ of the different samples were added and incubated at $37^{\circ} \mathrm{C}, 5 \% \mathrm{CO}_{2}$ for 24 and $48 \mathrm{~h}$. Then, $50 \mu \mathrm{L}$ of the supernatants were transferred to 96 -well microplates along with $50 \mu \mathrm{L}$ of the reaction mixture (Cytotoxicity Detection Kit (LDH), Roche, Basel, Switzerland) and left to react under orbital stirring (50 RPM), room temperature, and complete darkness for $15 \mathrm{~min}$. Absorbance was finally read at $490 \mathrm{~nm}$ in a microplate reader. The cytotoxicity was compared to that reported for magnetite in previous studies. 


\section{Results and Discussion}

\subsection{Morphology and Elemental Composition}

In order to investigate the size, size distribution, clustering, morphology, and silver shell coverage, TEM imaging was implemented. Figure 2A-C show the TEM images of patchy magnetite/silver nanoparticles. The individual patchy magnetite/silver particles' average size approaches $11.7 \mathrm{~nm}$, which agrees well with sizes reported elsewhere for similar coatings [32]. Arguably, the magnetite core's silver coating is unevenly distributed on the surface and is formed around clusters of magnetite nanoparticles, as it can be evidence from Figure 2B-C. These results agree well with those of Garza-Navarro et al., who also found a heterogeneous silver coverage [28]. In addition, the micrographs suggest that the coating varies from cluster to cluster.

Additionally, an SEM magnification was conducted (Figure 2E-F). The beam was focused on a cluster of nanoparticles, which shows the morphology of an agglomerate. There is an evident difference in size between the TEM and SEM results due to the magnification of each technique. While the TEM verifies the coating patchiness, SEM shows that there are regions with a major presence of silver, thereby suggesting marked differences in the patchiness between individual nanoparticles and clusters.

Besides inspecting the morphology, we conducted an Energy-dispersive X-ray spectroscopy (EDX) analysis of the nanoparticles (Figure 2D). The analyses confirmed the presence of iron and oxygen related to the magnetite core, silver, and chlorine from the shell, and possible $\mathrm{HCl}$ residues from bare magnetite nanoparticles' purification. The chlorine atoms on silver nanoparticles or bimetallic nanoparticles surface provide reactive sites for the subsequent conjugation of different macromolecules. For instance, $\mathrm{Hu}$ et al. conjugated a nitrile to the chlorine atoms on silver nanoparticles to label the HeLa cells' surface expressing a unique recombinant receptor [33]. The presence of silver and the chlorination extent were qualitatively assessed with the aid of the energy-dispersive spectroscopy (EDS) detector of the SEM instrument. The chlorine signal in Figure 2D-G corroborates chlorination's success and, therefore, the suitability for further functionalization [34]. The results also suggest that the purification process proved successful at separating bare nanoparticles of silver. This process is mainly enabled by the magnetite/silver's magnetic properties, which corroborated that the magnetism conferred by the magnetite core remained even after the silver coating. Additional studies are required to quantify the silver shell effect on magnetism.

\subsection{Magnetite/Silver Characterization}

Evidence provided by the UV-Vis data shown in Figure 3A supports the SEM and TEM results, thereby suggesting an incomplete or discontinuous coating of the silver shell. The silver shell presence was determined spectrophotometrically by collecting the absorbance in the $200-800 \mathrm{~cm}^{-1} \mathrm{range}_{\text {. }}$ Figure 3A shows the characteristic spectra of silver nanoparticles; the absorbance between 300 and $400 \mathrm{~cm}^{-1}$ increased compared to bare magnetite [22,32]. Still, the magnetite spectrum is visible in the range below $300 \mathrm{~cm}^{-1}$, indicating a discontinuous silver coverage [22,35]. Figure 3B presents the results of the hydrodynamic radii. Bare magnetite nanoparticles presented an average hydrodynamic diameter of $122 \mathrm{~nm}$ but increased to $295 \mathrm{~nm}$ after forming the silver shell. The corresponding polydispersity indexes (PI) increased from $22.6 \%$ to $43.5 \%$, which was comparable with those of magnetite nanoparticles previously used to conjugate an antimicrobial peptide [36,37]. A fascinating finding was the importance of the purification process toward the sample homogeneity, as evidenced by the UV-Vis spectra and the DLS data before and after purification. In this regard, Figure 3A-B (green) show data for the synthesized materials before purification. In the UV-Vis case, the observed absorbance is most likely due to the silver plasmon, as reported for similar materials elsewhere [24,32]. The particle size distribution shows that before purification, the sample exhibited significant clustering with particles that even reached the micron size range. Similar distributions have been observed previously for materials with different metal components due to different surface charge values [22,38]. 
The FTIR data in Figure 3A confirmed the purification process's effectiveness as peaks associated with organic compounds significantly decreased or even disappeared.

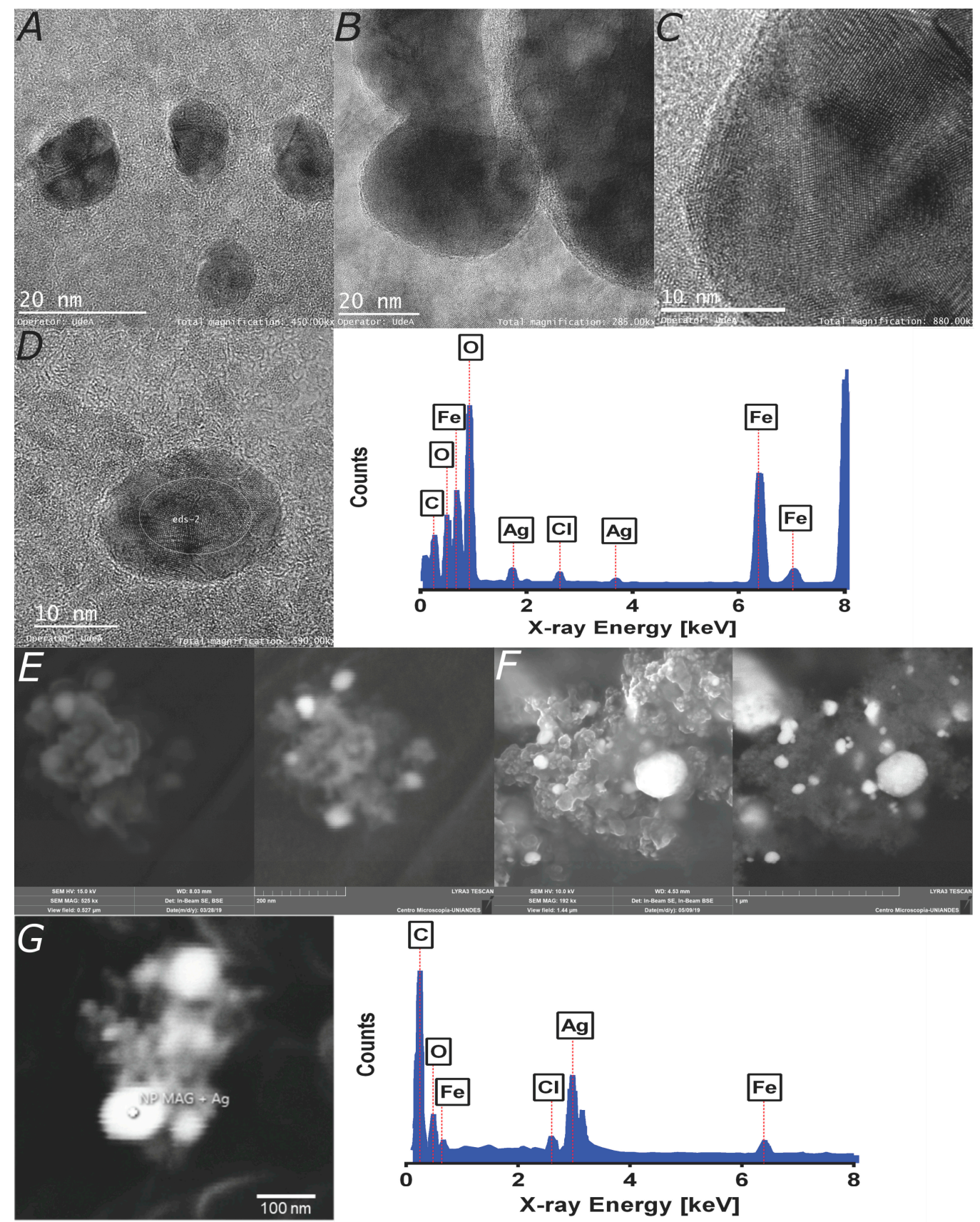

Figure 2. Microscopy imaging and elemental characterization of TEM images. (A) Magnetite/silver nanoparticles are exhibiting an average diameter of $11.7 \mathrm{~nm}$. (B) Magnetite clusters covered with a thin silver layer. (C) Patchy magnetite/silver nanoparticles as confirmed by the interplanar distances of the corresponding crystalline structures. (D) Elemental composition of the marked region, which confirms the presence of iron, silver, and chlorine. SEM images: (E) A core magnetite nanoparticles cluster synthesized by co-precipitation (scale bar corresponds to $200 \mathrm{~nm}$ ). (F) Patchy magnetite/silver nanoparticles show a definite change in morphology. White spots are most likely related to the silver coverage (scale bar corresponds to $1 \mu \mathrm{m}$ ). (G) The marked region's elemental composition verifies the presence of oxygen, iron, and silver, which are all related to the patchy bimetallic nanoparticles and chlorine remaining from the purification process. The carbon content is due to the graphite adhesive tape (scale bar corresponds to $100 \mathrm{~nm}$ ). 

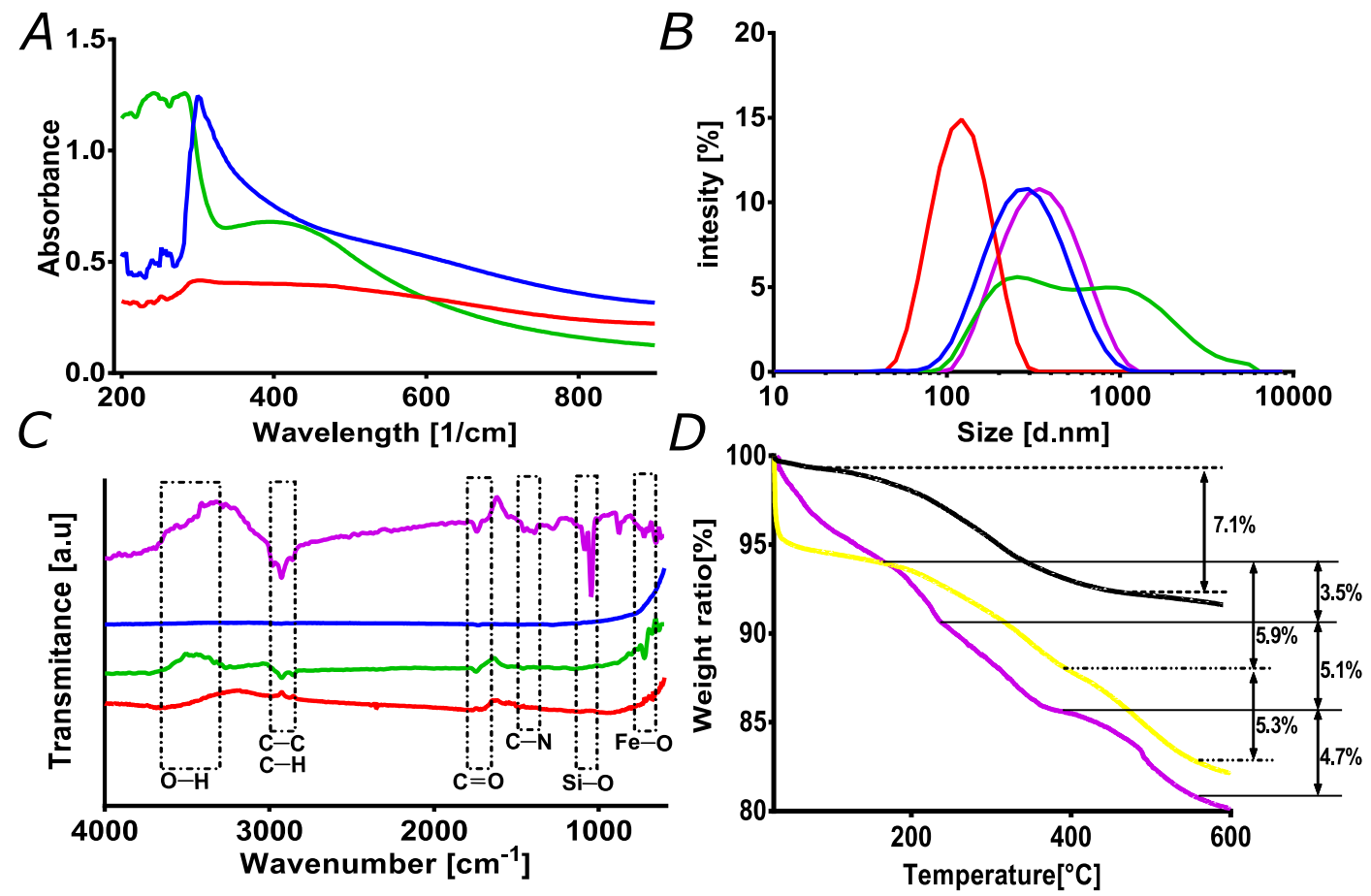

\section{Magnetite $\square$ Magnetite-APTES $\square$ Magnetite/Silver $\square$ Magnetite/Silver purified Magnetite/Silver-pDMAEMA Magnetite/Silver-pDMAEMA-APTES}

Figure 3. Characterization and purification process of magnetite/silver without purification (green). (A) UV-Visible spectra were collected to verify the silver coverage on magnetite nanoparticles, plus the effect of purification toward sample homogeneity. (B) Nanoparticles size distribution via dynamic light scattering. The data were useful to verify the homogeneity of the sample. (C) Fourier transform infrared spectroscopy (FTIR) spectra were used to evaluate the effectiveness of purification and cleaning of the nanoparticles and verify the presence of both chemistries on the magnetite/silver-pDMAEMA-APTES nanoparticles. Highlighted regions corresponding to pDMAEMA are $\mathrm{C}=\mathrm{O}, \mathrm{C}-\mathrm{C}$, and $\mathrm{C}-\mathrm{H}$ bonds. Simultaneously, the silanization is corroborated by Si-O and $\mathrm{C}-\mathrm{N}$ bonds, which are related to the conjugated pDMAEMA and APTES. The Fe-O bonds emerge from the magnetite core. (D) Thermogravimetric analyses (TGA) were also conducted to verify the effectiveness of conjugation for the magnetite-APTES, magnetite/silver-pDMAEMA, to magnetite/silver-pDMAEMA-APTES. APTES: (3-aminopropyl) triethoxysilane, pDMAEMA: poly(2-dimethylamino)ethyl methacrylate) methyl chloride quaternary salt.

The nanomaterials capable of withholding different chemistries on their surfaces, such as antibodies, small interfering RNA (siRNA), or peptides, are of great interest, particularly in the medical field [39,40]. In our case, we are interested in the conjugation of translocating peptides and proteins for efficient cell penetration and endosomal escape. After synthesis, we expected that our patchy bimetallic nanoparticles exhibited the surface of both metals exposed for further conjugation. This was tested by conjugating pDMAEMA to silver, which is a polymer used for cell penetration and endosomal escape thanks to its $\mathrm{pH}$-responsiveness. In this case, conjugation proceeded via Hoffman elimination and the chlorine chemistry [41,42]. As for the magnetite, the surface was evaluated by the conjugation of APTES, an organosilane molecule with an amine terminal that has been widely exploited for surface modification, as reported by Mohammed et al. in his review on magnetic nanoparticles in biomedical applications [40]. FTIR analyses were conducted to verify the successful conjugation by looking at the functional groups' presence from the conjugated molecules on the surface of the nanoparticles. Figure $3 \mathrm{C}$ compares the spectra of magnetite, magnetite/silver before purification, purified magnetite/silver, and the purified nanoparticles after conjugation with the APTES and pDMAEMA. The C-C and C-H 
stretching vibrations in the magnetite/silver-pDMAEMA nanoparticles spectrum at around $3390 \mathrm{~cm}^{-1}$ are related to the pDMAEMA structure. This is also the case for the peaks at 2857 and $2929 \mathrm{~cm}^{-1}$, corresponding to C-C's main chain and C-H stretching vibrations, respectively $[43,44]$. This result agrees well with similar characterizations for this polymer's conjugation by Liu et al. [44].

Moreover, the stretching vibration at $1740 \mathrm{~cm}^{-1}$ corresponds to the carbonyl $\mathrm{C}=\mathrm{O}$ group. At $1428 \mathrm{~cm}^{-1}$, the deformational stretching vibration of the $-\mathrm{N}\left(\mathrm{CH}_{3}\right)_{2}$ also belongs to the pDMAEMA $[35,43]$. Silanization, in this case, is the process by which the APTES binds covalently to the magnetite core of the patchy nanoparticles [45]. However, there exists a possibility for electrostatic interactions between the terminal amine group of APTES and the silver coating. We believe that, to a large extent, such interactions might be disrupted during the purification process by the thermal energy of hot water. Further studies are needed to estimate the relative fraction of adsorbed APTES compared with that covalently linked [46]. The peaks at 1065 and $950 \mathrm{~cm}^{-1}$ are for the Si-O-Si (due to silanization), and Fe-O bonds can be attributed to the magnetite core, respectively [30]. The silane chemistries on the nanomaterials' surface are widely used in the medical field for several applications ranging from delivering nucleic acids or detecting biological markers [35,39]. As previously discussed, one can highlight the importance of the purification process by evaluating DLS and UV-Vis results. As shown in Figure 3, the contrast of the blue and green plots demonstrate that bare nanoparticles' noise disappears in the UV-vis spectra, thereby displaying a well-defined peak when purified. The effectiveness of the process was corroborated by analyzing the extent of distribution of the hydrodynamic radii, where it is evident that the polydispersity of the sample decreases. Moreover, the FTIR analysis clearly shows the removal of organic compounds absorbed by the nanoparticles, as evidenced by a flat line representing the absence of major contaminants or impurities.

Thermogravimetric analyses were also conducted to estimate conjugation efficiencies (Figure 3D). With each sample's respective weight, all nanoparticles' samples exhibited an initial weight loss before $100{ }^{\circ} \mathrm{C}$, which is most likely due to the moisture adsorbed. As calculated from the weight loss, bare magnetite was conjugated with APTES with an efficiency of around $7.1 \%$. This result agrees well with previously reported studies [37]. Patchy magnetite/silver was conjugated, at first, with pDMAEMA, which showed a second weight loss of about $5.9 \%$. This is probably related to excess reagents adsorbed by the nanoparticles and the polymer. The third weight loss started at around $400{ }^{\circ} \mathrm{C}$ and was $5.3 \%$ of the total weight, which was most likely due to the polymer, as shown in studies involving the thermogravimetric analysis of pristine pDMAEMA [35,47]. Finally, the conjugation of both molecules (i.e., pDMAEMA and APTES) on the patchy magnetite/silver nanoparticles showed that the second weight loss approached $8.6 \%$, which can be attributed to the release of APTES and the adsorbed excess reagents on both the nanoparticles and the polymer, which was similar to that observed for the conjugation of the polymer. A final weight loss step above about $400{ }^{\circ} \mathrm{C}$ might be associated with the polymer pDMAEMA conjugated on the silver surface with $4.7 \%$ efficiency, which is comparable to previous reports [35,47]. Both FTIR and TGA confirmed the tunability of the surface of the patchy core/shell material synthesized, which was comparable to similar systems put forward previously in nanomedicine applications $[31,48]$. Thus, the patchy nanostructured material introduced here holds significant promise as an enabler of medical and biological applications. For instance, it is possible to conjugate individually or co-immobilize an ample range of biomolecules including DNA, RNA, antibodies, and recombinant proteins for applications ranging from sensing and diagnostics to highly targeted therapeutics in personalized medicine schemes. Moreover, it is possible to alter the properties of polymeric scaffolds for regenerative medicine to make them $\mathrm{pH}$-responsive and tunable by the inclusion of our patchy nanoparticles.

\subsection{Biocompatibility}

To evaluate the viability of the developed patchy magnetite/silver nanoparticles as potential nanoplatforms for biological applications such as drug delivery to treat different pathologies, it is crucial to study potential biocompatibility issues carefully. To achieve this, cytotoxicity, hemolytic, 
and platelet aggregation tendencies were evaluated. Figures $4 \mathrm{~A}$ and $4 \mathrm{~B}$ show the Vero cell viability after exposure to the magnetite/silver nanoparticles for 24 and $48 \mathrm{~h}$, respectively. Patchy magnetite/silver nanoparticles showed high cell viability levels of about $95 \%$, even after $48 \mathrm{~h}$ of incubation. Although silver is widely reported as a cytotoxic and genotoxic agent in mammalian cells [49], these results can be easily explained by the low silver content in the nanoparticles, as well as by the chemical interactions between the silver and the magnetite core that restrict the release of silver ions responsible for the reported cytotoxic activity [50]. Moreover, the obtained cell viability levels, compared to previously reported results of the cytocompatibility of magnetite nanoparticles (dotted line Figure 4A-B) [39], confirm that the silver shell shows no additional effect on the cytocompatibility.
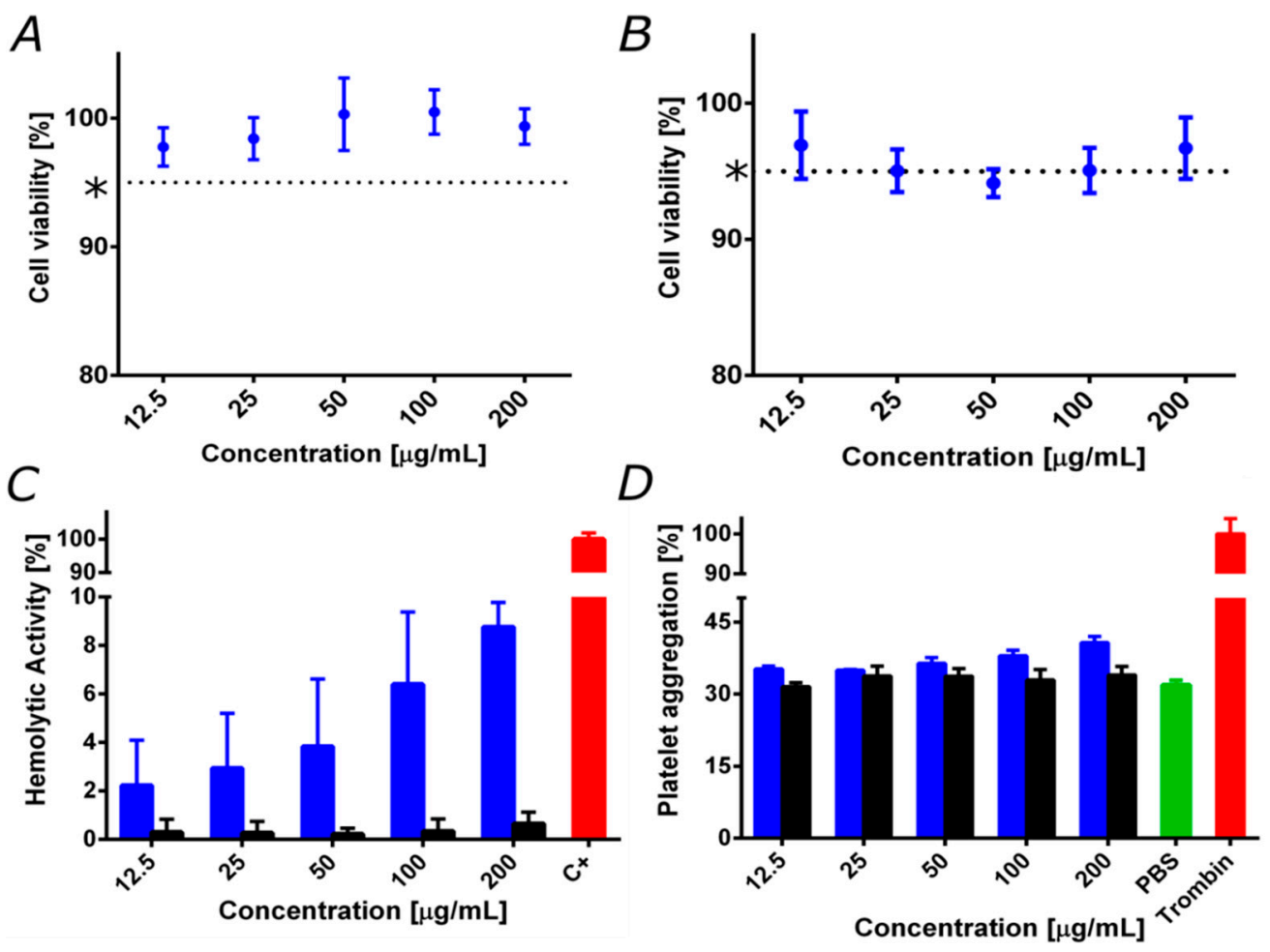

Figure 4. Biocompatibility assays for patchy magnetite/silver (blue) compared to bare magnetite (black). $\left(^{*}\right)$ magnetite cell viability reported elsewhere in the same $72 \mathrm{~h}$ and further [36,39]. (A) Cytotoxicity by lactate dehydrogenase enzyme (LDH) in Vero cells line after $24 \mathrm{~h}$. (B) Cytotoxicity by LDH in Vero cells after 48 h. Cell viability remained above $95 \%$. (C) The hemolysis assay shows an average hemolytic effect below $10 \%$ in all cases. The positive control was Triton 100-X. (D) Platelet aggregation assay shows an aggregation tendency of about $40 \%$, which is similar to that of bare magnetite. The negative and positive controls were PBS $1 \mathrm{X}$ and thrombin, respectively.

In addition, Figure $4 \mathrm{C}$ shows the hemolytic activity of the magnetite and the magnetite/silver nanoparticles. It is possible to observe that magnetite nanoparticles presented negligible hemolysis, as evidenced by an average percentage below $1 \%$ for concentrations up to $200 \mu \mathrm{g} / \mathrm{mL}$. In contrast, patchy magnetite/silver nanoparticles showed an average of $9 \%$ at the highest concentration $(200 \mu \mathrm{g} / \mathrm{mL})$. This result follows a typical dose-response tendency in which an increase in the concentration causes an increase in hemolytic activity. This agrees with previous studies that reported the high hemolytic effect for nanostructured silver [51].

Furthermore, Figure 4D shows the platelet aggregation tendency of the magnetite and the patchy magnetite/silver nanoparticles. Both types of nanoparticles presented a similar platelet aggregation percentage of about $30 \%$. However, it is possible to observe that an increase in the concentration of patchy magnetite/silver nanoparticles leads to an increase in the platelet aggregation percentage. 
This is consistent with previous articles that establish a relationship between the amount of silver and the platelet aggregation tendency [52]. The obtained results confirm the high biocompatibility of the synthesized patchy magnetite/silver nanoparticles in terms of low hemolytic and platelet aggregation tendencies and low LDH cytotoxicity. The superior biocompatibility obtained after purification is a critical feature to fully enable some of the applications described above. This is particularly important for complying with the increasingly stringent medical device regulatory frameworks emerging worldwide [1,53].

\section{Conclusions}

Patchy bimetallic nanoparticles instead of monometallic ones allow more versatile and tunable surface chemistries, which could be attractive to enable numerous biological and biomedical applications. Issues regarding environmental impact and reduced biocompatibility are still to be overcome to realize their full potential at a larger scale. Here, we introduced a green synthesis method to prepare patchy silver/magnetite nanoparticles to overcome such issues. This was with the final intention of putting forward a versatile platform to enable multifunctional nanostructures with high biocompatibility levels. This was achieved by a highly efficient downstream purification process that allowed removing excess toxic reagents and the target nanostructures' isolation. The success of each stage of the process was evaluated with the aid of UV-Vis, FTIR, and DLS. Successful conjugation of the $\mathrm{pH}$-responsive polymer pDMAEMA on the patchy silver shell and the organosilane APTES on the magnetite core confirmed the suitability of the nanostructures for highly efficient conjugation. Finally, the nanostructures exhibit high biocompatibility in terms of hemolysis, platelet aggregation, and LDH cytotoxicity. In conclusion, our study also provides details for the conjugation of multiples chemistries on the surface of the patchy bimetallic nanoparticles, which might be useful for emerging applications in nanomedicine where high biocompatibility is of the utmost importance.

Author Contributions: Conceptualization, J.C.C. and L.H.R.; methodology, J.C.C. and L.H.R.; validation, C.M.R.-A. and J.C.; formal analysis, C.M.R.-A., J.C., J.C.C. and L.H.R.; investigation, C.M.R.-A. and J.C.; resources, J.C.C. and L.H.R.; writing - original draft preparation, C.M.R.-A. and J.C.; writing-review and editing, J.C.C. and L.H.R.; visualization, C.M.R.-A. and J.C.; supervision, J.C.C. and L.H.R.; project administration, J.C.C. and L.H.R.; funding acquisition, J.C.C. and L.H.R. All authors have read and agreed to the published version of the manuscript.

Funding: This research was funded by the Colombian Ministry of Science, Technology, and Innovation (Minciencias), Grant ID 120380763212 - PPTA \# 8352. All authors acknowledge financial provided by the Vice Presidency for Research and Creation publication fund at the Universidad de los Andes.

Acknowledgments: We thank Humberto Ibarra, Monica Lopez, and Juan Camilo Orozco for performing the SEM + EDS and Confocal experiments. Johana Arboleda for TEM imaging. We like to acknowledge Johann Osma from the Electric and Electronics Engineering Department for inspiring discussions and additional insights into the characterization of our materials. Finally, the authors would like to thank the Departments of Chemical and Food Engineering, and Biomedical Engineering at Universidad de Los Andes for providing access to laboratory infrastructure.

Conflicts of Interest: The authors declare no conflict of interest.

\section{References}

1. Patra, J.K.; Das, G.; Fraceto, L.F.; Campos, E.V.R.; Rodriguez-Torres, M.D.P.; Acosta-Torres, L.S.; Diaz-Torres, L.A.; Grillo, R.; Swamy, M.K.; Sharma, S.; et al. Nano based drug delivery systems: Recent developments and future prospects. J. Nanobiotechnol. 2018, 16, 71. [CrossRef]

2. Varadan, V.K.; Chen, L.; Xie, J. Nanomedicine: Design and Applications of Magnetic Nanomaterials, Nanosensors and Nanosystems, 1st ed.; John Wiley \& Sons: Chichester, UK, 2008.

3. Lu, K.; Aung, T.; Guo, N.; Weichselbaum, R.; Lin, W. Nanoscale Metal-Organic Frameworks for Therapeutic, Imaging, and Sensing Applications. Adv. Mater. 2018, 30, 1707634. [CrossRef] [PubMed]

4. Medina-Cruz, D.; Saleh, B.; Vernet-Crua, A.; Nieto-Argüello, A.; Lomelí-Marroquín, D.; Vélez-Escamilla, L.Y.; Cholula-Díaz, J.L.; García-Martín, J.M.; Webster, T. Bimetallic nanoparticles for biomedical applications: A review. In Racing for the Surface: Antimicrobial and Interface Tissue Engineering; Springer International Publishing: Cham, Switzerland, 2020; pp. 397-434. ISBN 9783030344719. 
5. Srinoi, P.; Chen, Y.T.; Vittur, V.; Marquez, M.D.; Lee, T.R. Bimetallic nanoparticles: Enhanced magnetic and optical properties for emerging biological applications. Appl. Sci. 2018, 8, 1106. [CrossRef]

6. Gilroy, K.D.; Ruditskiy, A.; Peng, H.C.; Qin, D.; Xia, Y. Bimetallic nanocrystals: Syntheses, properties, and applications. Chem. Rev. 2016, 116, 10414-10472. [CrossRef]

7. Yamauchi, T.; Tsukahara, Y.; Yamada, K.; Sakata, T.; Wada, Y. Nucleation and growth of magnetic Ni?Co (Core?Shell) nanoparticles in a one-pot reaction under microwave irradiation. Chem. Mater. 2011, 23, 75-84. [CrossRef]

8. Unuofin, J.O.; Oladipo, A.O.; Msagati, T.A.M.; Lebelo, S.L.; Meddows-Taylor, S.; More, G.K. Novel silver-platinum bimetallic nanoalloy synthesized from Vernonia mespilifolia extract: Antioxidant, antimicrobial, and cytotoxic activities. Arab. J. Chem. 2020, 13, 6639-6648. [CrossRef]

9. Ma, W.F.; Zhang, Y.; Li, L.L.; You, L.J.; Zhang, P.; Zhang, Y.T.; Li, J.M.; Yu, M.; Guo, J.; Lu, H.J.; et al. Tailor-made magnetic $\mathrm{Fe}_{3} \mathrm{O}_{4} @ \mathrm{mTiO}_{2}$ microspheres with a tunable mesoporous anatase shell for highly selective and effective enrichment of phosphopeptides. ACS Nano 2012, 6, 3179-3188. [CrossRef]

10. Poon, Z.; Chen, S.; Engler, A.C.; Lee, H.; Atas, E.; von Maltzahn, G.; Bhatia, S.N.; Hammond, P.T. Ligand-Clustered "Patchy" Nanoparticles for Modulated Cellular Uptake and In Vivo Tumor Targeting. Angew. Chem. 2010, 122, 7424-7428. [CrossRef]

11. Khatami, M.; Alijani, H.Q.; Sharifi, I. Biosynthesis of bimetallic and core-shell nanoparticles: Their biomedical applications-A review. IET Nanobiotechnol. 2018, 12, 879-887. [CrossRef]

12. Geor malar, C.; Seenuvasan, M.; Kumar, K.S.; Kumar, A.; Parthiban, R. Review on surface modification of nanocarriers to overcome diffusion limitations: An enzyme immobilization aspect. Biochem. Eng. J. 2020, 158, 107574. [CrossRef]

13. Du, J.; Reilly, R.K.O. Anisotropic particles with patchy, multicompartment and Janus architectures: Preparation and application. Chem. Soc. Rev. 2011, 40, 2402-2416. [CrossRef]

14. Li, Y.; Bolinger, J.; Yu, Y.; Glass, Z.; Shi, N.; Yang, L.; Wang, M.; Xu, Q. Intracellular delivery and biodistribution study of CRISPR/Cas9 ribonucleoprotein loaded bioreducible lipidoid nanoparticles. Biomater. Sci. 2019, 7, 596-606. [CrossRef] [PubMed]

15. Yamada, Y.; Fukuda, Y.; Sasaki, D.; Maruyama, M.; Harashima, H. Development of a nanoparticle that releases nucleic acids in response to a mitochondrial environment. Mitochondrion 2020, 52, 67-74. [CrossRef]

16. Ealias, A.M.; Saravanakumar, M.P. A review on the classification, characterisation, synthesis of nanoparticles and their application. IOP Conf. Ser. Mater. Sci. Eng. 2017, 263, 32019.

17. Bianchi, E.; Capone, B.; Coluzza, I.; Rovigatti, L.; Van Oostrum, P.D.J. Limiting the valence: Advancements and new perspectives on patchy colloids, soft functionalized nanoparticles and biomolecules. Phys. Chem. Chem. Phys. 2017, 19, 19847-19868. [CrossRef] [PubMed]

18. Isaacoff, B.P.; Brown, K.A. Progress in Top-Down Control of Bottom-Up Assembly. Nano Lett. 2017, 17, 6508-6510. [CrossRef] [PubMed]

19. Lunn, D.J.; Finnegan, J.R.; Manners, I. Self-assembly of "patchy" nanoparticles: A versatile approach to functional hierarchical materials. Chem. Sci. 2015, 6, 3663-3673. [CrossRef]

20. Pawar, A.B.; Kretzschmar, I. Fabrication, assembly, and application of patchy particles. Macromol. Rapid Commun. 2010, 31, 150-168. [CrossRef]

21. Ravelo-Acuña, D.; Fuentes-García, J.A.; Yee-Madeira, H.T.; Diaz-Cano, A.I.; Goya, G.F.; Santoyo-Salazar, J. Sonochemical magnetite encapsulation in silica at low irradiation power. Mater. Lett. 2019, 250, $103-107$. [CrossRef]

22. Ghosh Chaudhuri, R.; Paria, S. Core/shell nanoparticles: Classes, properties, synthesis mechanisms, characterization, and applications. Chem. Rev. 2012, 112, 2373-2433. [CrossRef]

23. Tsai, T.H.; Thiagarajan, S.; Chen, S.M. Ionic liquid assisted one step green synthesis of Au-Ag bimetallic nanoparticles. J. Appl. Electrochem. 2010, 40, 493-497. [CrossRef]

24. Xia, L.; Zhang, F.; Liu, Z.; Ma, J.; Yang, J. Expression and characterization of cecropinXJ, a bioactive antimicrobial peptide from Bombyx mori (Bombycidae, Lepidoptera) in Escherichia coli. Exp. Ther. Med. 2013, 5, 1745-1751. [CrossRef] [PubMed]

25. Deplanche, K.; Merroun, M.L.; Casadesus, M.; Tran, D.T.; Mikheenko, I.P.; Bennett, J.A.; Zhu, J.; Jones, I.P.; Attard, G.A.; Wood, J.; et al. Microbial synthesis of core/shell gold/palladium nanoparticles for applications in green chemistry. J. R. Soc. Interface 2012, 9, 1705-1712. [CrossRef] [PubMed] 
26. Rodríguez-Fernández, D.; Liz-Marzán, L.M. Metallic Janus and patchy particles. Part. Part. Syst. Charact. 2013, 30, 46-60. [CrossRef]

27. Li, J.L.; Tian, B.; Li, T.; Dai, S.; Weng, Y.L.; Lu, J.J.; Xu, X.L.; Jin, Y.; Pang, R.J.; Hua, Y.J. Biosynthesis of Au, Ag and $\mathrm{Au}-\mathrm{Ag}$ bimetallic nanoparticles using protein extracts of deinococcus radiodurans and evaluation of their cytotoxicity. Int. J. Nanomed. 2018, 13, 1411-1424. [CrossRef]

28. Garza-Navarro, M.; Torres-Castro, A.; González, V.; Ortiz, U.; De la Rosa, E. Magnetite and magnetite/silver core/shell nanoparticles with diluted magnet-like behavior. J. Solid State Chem. 2010, 183, 99-104. [CrossRef]

29. Philip, D. Honey mediated green synthesis of silver nanoparticles. Spectrochim. Acta Part A Mol. Biomol. Spectrosc. 2010, 75, 1078-1081. [CrossRef]

30. Cheng, Z.; Tan, A.L.K.; Tao, Y.; Shan, D.; Ting, K.E.; Yin, X.J. Synthesis and characterization of iron oxide nanoparticles and applications in the removal of heavy metals from industrial wastewater. Int. J. Photoenergy 2012, 2012, 608298. [CrossRef]

31. Su, H.; Tian, Q.; Hurd Price, C.A.; Xu, L.; Qian, K.; Liu, J. Nanoporous core@shell particles: Design, preparation, applications in bioadsorption and biocatalysis. Nano Today 2020, 31, 100834. [CrossRef]

32. Dai, L.; Song, L.; Huang, Y.; Zhang, L.; Lu, X.; Zhang, J.; Chen, T. Bimetallic Au/Ag Core-Shell Superstructures with Tunable Surface Plasmon Resonance in the Near-Infrared Region and High Performance Surface-Enhanced Raman Scattering. Langmuir 2017, 33, 5378-5384. [CrossRef]

33. Hu, Q.; Tay, L.L.; Noestheden, M.; Pezacki, J.P. Mammalian cell surface imaging with nitrile-functionalized nanoprobes: Biophysical characterization of aggregation and polarization anisotropy in SERS imaging. J. Am. Chem. Soc. 2007, 129, 14-15. [CrossRef] [PubMed]

34. Stensberg, M.C.; Wei, Q.; McLamore, E.S.; Porterfield, D.M.; Wei, A.; Sepúlveda, M.S. Toxicological studies on silver nanoparticles: Challenges and opportunities in assessment, monitoring and imaging. Nanomedicine 2011, 6, 879-898. [CrossRef] [PubMed]

35. Ramírez-acosta, C.M.; Cifuentes, J.; Castellanos, M.C.; Moreno, R.J.; Muñoz-camargo, C.; Cruz, J.C.; Reyes, L.H. Ph-responsive, cell-penetrating, core/shell magnetite/silver nanoparticles for the delivery of plasmids: Preparation, characterization, and preliminary in vitro evaluation. Pharmaceutics 2020, $12,561$. [CrossRef] [PubMed]

36. Cuellar, M.; Cifuentes, J.; Cruz, J.; Perez, J.; Suarez-Arnedo, A.; Muñoz-Camargo, C.; Serna, J.; Groot, H. Novel BUF2-magnetite nanobioconjugates with cell-penetrating abilities. Int. J. Nanomed. 2018, 13, 8087-8094. [CrossRef]

37. Perez, J.; Cifuentes, J.; Cuellar, M.; Suarez-Arnedo, A.; Cruz, J.C.; Muñoz-Camargo, C. Cell-Penetrating And Antibacterial BUF-II Nanobioconjugates: Enhanced Potency Via Immobilization On Polyetheramine-Modified Magnetite Nanoparticles. Int. J. Nanomed. 2019, 14, 8483-8497. [CrossRef]

38. Matahum, J.S.; Su, C.M.; Wang, W.J.; Lou, S.L.; Ger, T.R. Effect of Surface Charge on the Uptake of Magnetic Nanoparticles in Mouse Fibroblast Cells. IEEE Magn. Lett. 2017, 8, 1-5. [CrossRef]

39. Lopez-Barbosa, N.; Garcia, J.G.; Cifuentes, J.; Castro, L.M.; Vargas, F.; Ostos, C.; Cardona-Gomez, G.P.; Hernandez, A.M.; Cruz, J.C. Multifunctional magnetite nanoparticles to enable delivery of siRNA for the potential treatment of Alzheimer's. Drug Deliv. 2020, 27, 864-875. [CrossRef]

40. Mohammed, L.; Gomaa, H.G.; Ragab, D.; Zhu, J. Magnetic nanoparticles for environmental and biomedical applications: A review. Particuology 2017, 30,1-14. [CrossRef]

41. Fan, X.X.; Xie, R.; Zhao, Q.; Li, X.Y.; Ju, X.J.; Wang, W.; Liu, Z.; Chu, L.Y. Dual pH-responsive smart gating membranes. J. Memb. Sci. 2018, 555, 20-29. [CrossRef]

42. You, Y.Z.; Manickam, D.S.; Zhou, Q.H.; Oupický, D. Reducible poly(2-dimethylaminoethyl methacrylate): Synthesis, cytotoxicity, and gene delivery activity. J. Control. Release 2007, 122, 217-225. [CrossRef]

43. Majewski, A.P.; Schallon, A.; Jérôme, V.; Freitag, R.; Müller, A.H.E.; Schmalz, H. Dual-responsive magnetic core-shell nanoparticles for nonviral gene delivery and cell separation. Biomacromolecules 2012, 13, 857-866. [CrossRef]

44. Liu, X.; Ni, P.; He, J.; Zhang, M. Synthesis and micellization of $\mathrm{pH} /$ temperature-responsive double-hydrophilic diblock copolymers polyphosphoester- block -poly[2-(dimethylamino)ethyl methacrylate] prepared via ROP and ATRP. Macromolecules 2010, 43, 4771-4781. [CrossRef]

45. Ghutepatil, P.R.; Salunkhe, A.B.; Khot, V.M.; Pawar, S.H. APTES (3-aminopropyltriethoxy silane) functionalized $\mathrm{MnFe}_{2} \mathrm{O}_{4}$ nanoparticles: A potential material for magnetic fluid hyperthermia. Chem. Pap. 2019, 73, 2189-2197. [CrossRef] 
46. Jaramillo, A.F.; Baez-Cruz, R.; Montoya, L.F.; Medinam, C.; Pérez-Tijerina, E.; Salazar, F.; Rojas, D.; Melendrez, M.F. Estimation of the surface interaction mechanism of $\mathrm{ZnO}$ nanoparticles modified with organosilane groups by Raman Spectroscopy. Ceram. Int. 2017, 43, 11838-11847. [CrossRef]

47. Meyer, F.; Minoia, A.; Raquez, J.M.; Spasova, M.; Lazzaroni, R.; Dubois, P. Poly(amino-methacrylate) as versatile agent for carbon nanotube dispersion: An experimental, theoretical and application study. J. Mater. Chem. 2010, 20, 6873-6880. [CrossRef]

48. Sperling, L.E.; Reis, K.P.; Pranke, P.; Wendorff, J.H. Advantages and challenges offered by biofunctional core-shell fiber systems for tissue engineering and drug delivery. Drug Discov. Today 2016, 21, 1243-1256. [CrossRef]

49. Setyawati, M.I.; Yuan, X.; Xie, J.; Leong, D.T. The influence of lysosomal stability of silver nanomaterials on their toxicity to human cells. Biomaterials 2014, 35, 6707-6715. [CrossRef]

50. Sanyasi, S.; Majhi, R.K.; Kumar, S.; Mishra, M.; Ghosh, A.; Suar, M.; Satyam, P.V.; Mohapatra, H.; Goswami, C.; Goswami, L. Polysaccharide-capped silver Nanoparticles inhibit biofilm formation and eliminate multi-drug-resistant bacteria by disrupting bacterial cytoskeleton with reduced cytotoxicity towards mammalian cells. Sci. Rep. 2016, 6, 24929. [CrossRef]

51. Choi, J.; Reipa, V.; Hitchins, V.M.; Goering, P.L.; Malinauskas, R.A. Physicochemical Characterization and in vitro hemolysis evaluation of silver nanoparticles. Toxicol. Sci. 2011, 123, 133-143. [CrossRef]

52. Jun, E.A.; Lim, K.M.; Kim, K.; Bae, O.N.; Noh, J.Y.; Chung, K.H.; Chung, J.H. Silver nanoparticles enhance thrombus formation through increased platelet aggregation and procoagulant activity. Nanotoxicology 2011, 5, 157-167. [CrossRef]

53. Kumar, R.; Mondal, K.; Panda, P.K.; Kaushik, A.; Abolhassani, R.; Ahuja, R.; Rubahn, H.-G.; Mishra, Y.K. Core-Shell Nanostructures: Perspectives towards Drug Delivery Applications. J. Mater. Chem. B 2020. [CrossRef] [PubMed]

(C) 2020 by the authors. Licensee MDPI, Basel, Switzerland. This article is an open access article distributed under the terms and conditions of the Creative Commons Attribution (CC BY) license (http://creativecommons.org/licenses/by/4.0/). 